
\title{
.
}

\section{Role of Continuous High Thoracic Epidural Anesthesia in Hippocampal Apoptosis after Global Cerebral Ischemia in Rats}

\author{
Xuan Li ${ }^{a}$ Xing Huo Chongyou Zhang ${ }^{\mathrm{b}}$ Xinyu Ma $\mathrm{Ma}^{\mathrm{a}, \mathrm{c}}$ Fei Han ${ }^{\mathrm{a}}$ Guonian Wang \\ aDepartment of Anesthesiology, Cancer Hospital and Pain Institute of Harbin Medical University, \\ Harbin, ${ }^{b}$ College of Basic Medicine, Harbin Medical University, Harbin, 'Department of Anesthesiology, \\ Affiliated Hospital of Chifeng University, Chifeng, Nei Monggol, China
}

\section{Key Words}

High thoracic epidural anesthesia (HTEA) - Cerebral ischemia - Global • Rats - Apoptosis • Poly (ADP-ribose) polymerase (PARP)

\begin{abstract}
Background: Cervical sympathetic blockade has been found to reduce cerebral vascular resistance and improve focal cerebral ischemia/reperfusion injury. In this study, we tested the hypothesis that the sympathetic blockade of high thoracic epidural anesthesia (HTEA) would reduce hippocampal apoptosis after global cerebral ischemia (GCI) injury. Methods: Fifteen-minute global ischemia was established by 4-vessel occlusion in adult male Wistar rats. And $0.5 \%$ bupivacaine or $0.9 \%$ saline $(20 \mu \mathrm{l} / / \mathrm{h})$ was infused continuously to the thoracic epidural space through the T4-5 intervertebral space from 15 minutes before ischemia to 24 hours or 72 hours after ischemia. Cerebral blood flow (CBF), Mortality, neurodeficit scores (NDS), Nissl and TUNEL staining, hippocampal superoxide dismutase (SOD) activity, malondialdehyde (MDA) concentrations, western blot of poly (ADP-ribose) polymerase (PARP) and immunohistochemical staining (PARP, Bax and $\mathrm{Bcl}-2$ ) were determined. Results: Both the hyperpefusion and hypoperfusion after reperfusion were improved by HTEA. HTEA decreased the number of apoptotic neurons in cornu ammonis area 1 (CA1), reduced PARP and Bax expressions with a decrease of $\mathrm{Bax} / \mathrm{Bcl}-2$ ratio induced by ischemic injury. The upregulation of SOD activity and the downregulation of MDA were obvious in the HTEA group compared with the GCI group. HTEA also improved NDS but not the mortality rate. Conclusion: Our study demonstrated that continuous HTEA attenuates hippocampal apoptosis and a behavioral deficit after global cerebral ischemia, and that these protective effects are associated with the improved microcirculation, reduced oxidative stress and the less activation of PARP.
\end{abstract}

X. Li and X. Huo contributed equally to this work. 


\section{Introduction}

Global cerebral ischemia (GCI) is a clinical outcome occurring as a consequence of conditions like cardiac arrest, coronary artery bypass surgery and reversible severe hypotension [1]. As for cerebral ischemic injury, it is generally accepted that apoptosis plays a pivotal role in cell death after hypoxia [2]. Pyramidal neurons in cornu ammonis area 1 (CA1) of the hippocampus are selectively vulnerable to GCI, and it has been suggested that the delayed neuronal death 3 or 4 days after ischemic insult is apoptosis [3].

Poly (ADP-ribose) polymerase (PARP) is a tightly bound nuclear enzyme found in various organs, including the brain. PARP is thought to play a physiological role in maintaining genomic integrity and in the repair of DNA strand breaks, and it is also thought to participate in cellular differentiation, gene rearrangement and transpositions during cerebral ischemia [4]. In global ischemia/reperfusion model, oxygen radicals initiate the mitochondrial pathway of apoptosis with the release of mitochondrial cytochrome $c$ to the cytoplasm, followed by the activation of the cytochrome $c$-dependent caspase cascade. The downstream caspases cleave many substrate proteins, including PARP [2]. PARP proteolytic cleavage provides an important early marker for apoptosis, and it might be directly responsible for the characteristic changes associated with apoptosis [5]. PARP activation and DNA damage mainly mediated by reactive oxygen species (ROS) and nitric oxide [4], which are provided by reoxygenation during reperfusion, and scavenged by superoxide dismutase (SOD) [6]. ROS also induce malondialdehyde (MDA) which is an indicator of lipid peroxidation [7].

In the model of global cerebral ischemia-reperfused animals, there is a period of hyperperfusion followed by a period of hypoperfusion. During reperfusion, capillary flow velocities, precapillary arteriole diameters and capillary diameters were decreased, which indicate that delayed hypoperfusion occurs as a consequence of increased precapillary arteriole tone $[8,9]$. The increased microvascular resistance and impaired reperfusion could be responsible for ultimate neuronal death, rather than the period of global ischemic hypoxia [8], and the post-ischemic hypoperfusion can be ameliorated by reducing vascular tone.

The cerebral vasculature, including that of the ventral hippocampus receives a sympathetic nerve supply $[10,11]$. The sympathetic nerve distribution to the head, enabling and mediating vasomotor responsiveness, stems from the 3 ganglia of the cervical sympathetic chain, which originate in the upper thoracic segments $[12,13]$. Thoracic sympathectomy as well as stellate ganglion block (SGB) increased the blood flow volume, rate and the vascular diameter in the common carotid artery of the patients $[14,15]$. SGB reduced ipsilateral cerebral vascular resistance, improve brain perfusion and oxygen saturation in patients with chronic ischemic stroke or pain $[14,15]$, and cervical sympathetic block can relieve delayed cerebral ischemia after subarachnoid hemorrhage (SAH) in human and rabbits [16, 17], but the intracranial vessels were unaffected by SGB in healthy volunteers [18]. Recent studies revealed that SGB and sympathetic ganglionectomy have little influence on cerebral autoregulation under normal physiological conditions, but it is of importance that sympathetic control of cerebral blood flow (CBF) decreases sympathetic tone under pathologic conditions $[19,20]$. Furthermore, focal cerebral ischemia/reperfusion injury of rats could be improved under SGB and cervical sympathetic ganglionectomy by expanding cerebral vessels and regulating cytokine release [21].

High thoracic epidural anesthesia (HTEA) maintained with local anesthetic agents, is usually used to overcome intraopertive and postoperative pain after surgery. In addition, TEA induces preganglionic sympathetic blockade, and has been suggested to offer protective cardiac, pulmonary and gastrointestinal effects as well as positive immunological and coagulation properties [22]. But little is known about the relationship between HTEA and cerebral ischemia.

The present study was designed to investigate the neurological and morphological changes in response to HTEA in the hippocampus of rat with GCI. Furthermore, to explore the mechanism we also determined whether the disturbance of CBF, the reduced level of lipid peroxidation and oxygen free radicals, and the reduced activation of PARP are involved. 


\section{Materials and Methods}

\section{Animals and Surgical Procedures}

This study was approved by the Harbin Medical University Animal Care and Use Committee in Harbin, China. Eighty-seven adult male Wistar rats, weighing 280 to 320 g, were divided into 4 groups: sham (n=22), sham-HTEA $(n=4)$, GCI $(n=30)$, HTEA $(n=31)$. The animals were obtained from the animal experiment center at the Second Affiliated Hospital of Harbin Medical University, acclimatized for at least 3 days before the experiments, had free access to food and tap water and were maintained at $21 \pm 2{ }^{\circ} \mathrm{C}$ for a $12 \mathrm{~h}$ dark and light cycle.

After injection of $10 \%$ chloral hydrate $(300 \mathrm{mg} / \mathrm{kg}$, i.p.), epidural catheterization was performed, using the method previously described [23]. Each rat was placed in the prone position. The ligaments between the intervertebral spaces (T4 to 5) were severed to visualize the white dura. Epidural catheters (A PE10 tube with a $0.61 \mathrm{~mm}$ outer diameter) were inserted into the epidural space and threaded cephalad approximately 10-15 mm. A repeated negative liquor aspiration test excluded subdural positioning of the catheter. The epidural catheter was fixed, tunneled under the skin and protected by a metal swivel. This procedure allowed for continuous infusion and for unrestricted animal movement within its individual cage. A successful implantation of epidural catheter was testified after the animal was immediately recovered from the surgery and anesthesia by detection of reversible loss of pain sensation in front limbs and the segmental thoracic area (T1-T4) without motor and sensory disturbance in hind limbs following injection of $20 \mu \mathrm{l}$ of $1 \%$ lidocaine through the catheter [23]. After completion of the experiment, the position of the catheter was verified by autopsy.

Global cerebral ischemia in rats was inflicted according to the 4-vessel occlusion (4-V0) method [24]. Briefly, the rat vertebral arteries were electrocoagulated through the alar foramen of the first cervical vertebra on the stereotaxic frame after epidural catheterization. Twenty-four hours later, awake rats were anesthetized with chloral hydrate, and both carotid arteries were then clamped by atraumatic arterial clasps to cause vascular occlusion. And the standard of satisfactory cerebral ischemia was that the electroencephalography (EEG) became isoelectric within 2-3 min and remained isoelectric throughout the ischemic period. During the course of performing vascular occlusion, the animals remained unconscious and indicated no signs of pain or suffering [25]. After $15 \mathrm{~min}$ of ischemia, the atraumatic arterial clasps were removed to permit reperfusion. During ischemia, the left femoral artery and venous were cannulated, mean arterial blood pressure (MAP), heart rate (HR) and EEG were monitored with a multifunction physiological signal acquisition and processing system (RM6240, Chengdu, China). Arterial blood was sampled anaerobically, and $\mathrm{PaO}_{2}, \mathrm{PaCO}_{2}$ and $\mathrm{pH}$ were measured with a blood gas analyzer (Bayer Diagnostics, Norwood, MA). Saline solution $2 \mathrm{~mL} / \mathrm{h}$ was infused throughout the complete observational period. Epidural infusion started as continuous infusion $15 \mathrm{~min}$ before ischemia [23] and continued until the end of the reperfusion period ( 24 or 72 hours) [7]. Rectal temperature was maintained at a level of $37.0{ }^{\circ} \mathrm{C} \pm 0.5{ }^{\circ} \mathrm{C}$ with a heating pad. After $2 \mathrm{~h}$ of reperfusion, temperature probes, needle electrodes, and vessel catheters were removed, incisions were closed, and the animals were allowed to wake up in its individual cage. Free access to food and water was then allowed.

\section{Measurement of $C B F$}

Sixteen rats with successfully developed high thoracic epidural catheterization and global cerebral ischemia were randomly assigned to four groups $(\mathrm{n}=4)$ : Sham-sham procedure, $20 \mu \mathrm{l} / \mathrm{h} \mathrm{NaCl}, 0.9 \%$, epidural; Sham +HTEA-sham procedure, $20 \mu \mathrm{l} / \mathrm{h}$ bupivacaine, 0.5\%, epidural; GCI- 4-VO, $20 \mu \mathrm{l} / \mathrm{h} \mathrm{NaCl}$, $0.9 \%$, epidural; or HTEA - 4-VO, $20 \mu \mathrm{l} / \mathrm{h}$ bupivacaine, $0.5 \%$, epidural. The sham group had epidural catheterization, alar foramen and the common carotid arteries exposed without vascular occlusion. Cerebral blood flow (CBF) was measured continuously with a laser Doppler flowmeter (Periflux System 5010, Perimed, Sweden) as described previously [26]. After the scalp was opened with a scalpel, the probe holder containing a LDF probe was attached to the right skull ( $5 \mathrm{~mm}$ lateral and $1 \mathrm{~mm}$ posterior to the bregma). CBF was measured 2 minutes before both common arteries were occluded as the baseline value, at the time of ischemia and 0 to 120 minutes after reperfusion. CBF values were expressed as percentages relative to baseline $(100 \%)$. 


\section{Neurons Counts and Immunohistochemistry}

An additional 29 animals were randomly divided into 3 groups: a sham-operated group ( $n=9)$; a GCI group ( $n=11)$; and a HTEA group $(n=9)$. The rats were transcardially perfused with 200 ml ice-cold saline and subsequently with 4\% paraformaldehyde in PBS 24 or 72 hours after reperfusion. The brain was removed, postfixed for 24 hours in paraformaldehyde solution. After dehydration in gradient sucrose, the coronal sections of brain were prepared at the level of $3.3 \pm 0.2 \mathrm{~mm}$ posterior to bregma and stored at $-80{ }^{\circ} \mathrm{C}$. Frozen sections $(10 \mu \mathrm{m})$ at 72 hours after reperfusion ( $\mathrm{n}=4$ per group) were used for Nissl and TUNEL staining. They were dried at room temperature, rehydrated, and immersed in warmed $0.5 \%$ cresyl violet solution (10 minutes). Neuronal apoptosis in the hippocampal CA1 region was assessed by TUNEL staining, using an in situ cell death detection kit (TMR green, Roche, Mannheim, Germany). The brain sections at 24 hours after reperfusion (sham, $n=5$; GCI, $n=7$; HTEA, n=5) were used to perform immunohistochemistry as described [27]. The primary antibodies were: rabbit polyclonal antibody against PARP $(1: 200$, ab6079, Abcam), Bcl-2 (1:100, BA0412, Boster) or Bax (1:100, BA0315-1, Boster).

Six photomicrographs of both hemispheres hippocampal CA1 layers were taken from each animal. The mean numbers of neurons were expressed as the number of surviving neurons and TUNEL-positive cells per millimeter linear length in the CA1 region of the dorsal hippocampus as described earlier [28, 29]. The PARP positive cells were expressed as an average fraction of the total number of neurons. The Image Pro Plus software, version 6.0 (Media Cybernetics, USA) was used to analyze the average optical density (OD) of Bcl-2 and Bax. An investigator who was blinded to the experimental conditions made all assessments at 400 -fold magnification.

\section{Western Blot Analysis}

Another 18 animals of the above 3 groups were sacrificed at 24 or $72 \mathrm{~h}$ after ischemia ( $\mathrm{n}=3$ per group). The brain was quickly removed and the hippocampal tissue was rapidly isolated. Western blot analysis was performed as described previously [28]. The primary antibodies were: rabbit anti-PARP polyclonal antibody (1:1000, \#9542, Cell Signaling Technology) and anti- $\beta$-actin (1:1000, M1501, HaiGene, China). The secondary antibody was goat anti-rabbit IgG antibody (1:5000, M0201, HaiGene, China).

\section{Measurement of SOD Activity and MDA Concentration}

The homogenized hippocampal tissue at $24 \mathrm{~h}$ after reperfusion ( $\mathrm{n}=6$ per group) was centrifuged (3000 $\mathrm{g} \times 10 \mathrm{~min}$ ), and the supernatant was collected to measure MDA content and SOD activity. MDA and SOD levels were measured using a commercial kit (Jiancheng Biochemistry Co., Nanjing, China) at $532 \mathrm{~nm}$ by the thiobarbituric acid method, and at $550 \mathrm{~nm}$ by the xanthine oxidase method, respectively.

\section{Neurological Evaluation}

The Neurodeficit Score (NDS) of all the animals was evaluated at $24 \mathrm{~h}$ (sham, n=11; GCI, n=13; HTEA, $\mathrm{n}=11$ ) and $72 \mathrm{~h}$ ( $\mathrm{n}=7$ per group) after GCI according to previous studies [30]. In brief, scoring includes seven parameters: general behavior, brain stem function, motor and sensory function assessment, motor behavior (including gait coordination and balance on a beam), other behavior reflexes, and seizures. An NDS of 80 reflected normal brain function, whereas an NDS of 0 represented brain death. The evaluation was always carried out by the same investigator who was blinded to the experimental groups.

\section{Statistical Analysis}

Data are presented as the mean \pm SD, one-way ANOVA and Student-Newman-Keuls test for post hoc comparisons was used to verify the significance of intergroup differences in PARP positive percent, Bax and Bcl-2 OD and the ratio, NDS, SOD activity and MDA level. Fisher's exact test was used for analyzing mortality, repeated measures analysis of variance for physiological variables and CBF, a non-parametric Kruskal-Wallis analysis for the cell count. A $P$-value of $P<0.05$ was considered significant.

\section{Results}

Physiological Variables and CBF by Laser Doppler during Ischemia and Reperfusion Body weight and rectal temperatures during the ischemia/reperfusion periods showed no significant difference among the experimental groups. Analyzing MAP, HR and blood 
Table 1. Physiological variables and blood gases during Ischemia and Reperfusion. MAP: mean blood pressure; $\mathrm{HR}=$ heart rate; $\mathrm{PaO}_{2}$ : arterial oxygen tension; $\mathrm{PaCO}_{2}$ : arterial carbon dioxide tension. HTEA: high thoracic epidural anesthesia; GCI: global cerebral ischemia. ${ }^{*} P<0.05$, compared with sham group

\begin{tabular}{|c|c|c|c|c|c|c|c|}
\hline & & Pre-Ischemia & Ischemia 0min & Ischemia $5 \mathrm{~min}$ & $\begin{array}{l}\text { Ischemia } \\
10 \mathrm{~min}\end{array}$ & $\begin{array}{c}\text { Reperfusion } \\
\text { Omin }\end{array}$ & $\begin{array}{c}\text { Reperfusion } \\
15 \mathrm{~min}\end{array}$ \\
\hline \multirow[t]{4}{*}{ MAP (mmHg) } & Sham & $107.4 \pm 7.48$ & $111.5 \pm 5.78$ & $113 \pm 5.81$ & $113.9 \pm 5.02$ & $117.5 \pm 5.45$ & $115.9 \pm 6.03$ \\
\hline & Sham-HTEA & $100.7 \pm 5.79$ & $92.3 \pm 7.23$ & $99.5 \pm 8.54$ & $91.8 \pm 5.19$ & $109.3 \pm 8.46$ & $99.3 \pm 7.27$ \\
\hline & GCI & $100.7 \pm 15.27$ & $132.4 \pm 23.68^{*}$ & $122.4 \pm 18.91$ & $114.4 \pm 19.5$ & $95.7 \pm 13.48^{*}$ & $104.8 \pm 17.26$ \\
\hline & HTEA & $98.2 \pm 14.74$ & $131.0 \pm 18.94^{*}$ & $120.1 \pm 15.33$ & $111.1 \pm 9.45$ & $94.4 \pm 19.53^{*}$ & $101.6 \pm 14.49$ \\
\hline \multirow[t]{4}{*}{ HR (beats/min) } & Sham & $400.9 \pm 28.83$ & $401.8 \pm 27.94$ & $403.6 \pm 28.57$ & $403.9 \pm 8.36$ & $407.4 \pm 30.46$ & $406.3 \pm 25.61$ \\
\hline & Sham-HTEA & $407.2 \pm 36.52$ & $398.4 \pm 28.46$ & $389.7 \pm 35.84$ & $398.6 \pm 7.94$ & $384.9 \pm 38.42$ & $394.2 \pm 34.93$ \\
\hline & GCI & $411.2 \pm 48.76$ & $432.2 \pm 49.19$ & $387.1 \pm 56.01$ & $395.4 \pm 62.74$ & $411.4 \pm 64.54$ & $415.3 \pm 46.11$ \\
\hline & HTEA & $383.2 \pm 44.07$ & $412.5 \pm 64.77$ & $383.8 \pm 54.99$ & $397.4 \pm 60.59$ & $388.9 \pm 74.75$ & $383.1 \pm 54.63$ \\
\hline \multirow[t]{4}{*}{$\mathrm{PaCO} 2(\mathrm{mmHg})$} & Sham & $35.5 \pm 3.62$ & & $34.7 \pm 3.72$ & & $34.7 \pm 3.56$ & $34.0 \pm 2.61$ \\
\hline & Sham-HTEA & $33.8 \pm 5.85$ & & $34.3 \pm 5.20$ & & $37.5 \pm 4.87$ & $35.7 \pm 4.83$ \\
\hline & $\mathrm{GCI}$ & $30.7 \pm 3.78$ & & $24.3 \pm 1.97^{*}$ & & $20.5 \pm 1.87^{*}$ & $27.8 \pm 2.42^{*}$ \\
\hline & HTEA & $31.4 \pm 4.25$ & & $25.5 \pm 2.73^{*}$ & & $22.1 \pm 3.63^{*}$ & $28.3 \pm 2.07^{*}$ \\
\hline \multirow[t]{4}{*}{ Pa02 (mmHg) } & Sham & $92.5 \pm 1.87$ & & $93.7 \pm 2.73$ & & $94.0 \pm 3.74$ & $94.3 \pm 1.75$ \\
\hline & Sham-HTEA & $91.4 \pm 5.82$ & & $95.2 \pm 6.84$ & & $94.1 \pm 8.34$ & $93.6 \pm 3.68$ \\
\hline & GCI & $89.5 \pm 2.59$ & & $104.7 \pm 5.85^{*}$ & & $98.7 \pm 3.61$ & $92.5 \pm 2.07$ \\
\hline & HTEA & $88.8 \pm 4.84$ & & $104.4 \pm 9.25^{*}$ & & $95.5 \pm 2.07$ & $93.0 \pm 3.71$ \\
\hline \multirow[t]{4}{*}{ Arterial $\mathrm{pH}$} & Sham & $7.41 \pm 0.03$ & & $7.42 \pm 0.05$ & & $7.40 \pm 0.03$ & $7.40 \pm 0.03$ \\
\hline & Sham-HTEA & $7.46 \pm 0.04$ & & $7.44 \pm 0.07$ & & $7.46 \pm 0.05$ & $7.45 \pm 0.04$ \\
\hline & $\mathrm{GCI}$ & $7.44 \pm 0.02$ & & $7.54 \pm 0.05^{*}$ & & $7.57 \pm 0.03^{*}$ & $7.45 \pm 0.08$ \\
\hline & HTEA & $7.43 \pm 0.02$ & & $7.53 \pm 0.06^{*}$ & & $7.56 \pm 0.06^{*}$ & $7.44 \pm 0.04$ \\
\hline
\end{tabular}

gas parameters, neither the difference between sham and sham-HTEA groups, nor that between GCI and HTEA groups was significant (Table 1). MAP rose immediately (ischemia 0 $\mathrm{min}$ ) after bilateral carotid artery ligation, and decreased immediately (reperfusion $0 \mathrm{~min}$ ) after reperfusion in both GCI and HTEA groups compared with the sham group $(P<0.05)$. Four-vessel occlusion resulted in hyperventilation with an elevation of $\mathrm{PaO}_{2}$ and $\mathrm{pH}$, and a decrease of $\mathrm{PaCO}_{2}(P<0.05)$. And $\mathrm{MAP} \mathrm{PaO}_{2}$ and $\mathrm{pH}$ returned to sham values within 15 min of carotid clasp removal. The value of $\mathrm{PaCO}_{2}$ in both GCI and HTEA groups remained lower than that of the sham group at $15 \mathrm{~min}$ after reperfusion $(P<0.05)$.

Throughout the experiment, there were no significant changes of CBF in the sham group and sham-HTEA group animals (Fig. 1). CBF was reduced to $9.66 \pm 2.56 \%$ in GCI group and $21.74 \pm 9.48 \%$ in HTEA group during global cerebral ischemia. After 10 minutes of reperfusion, the CBF of hyperperfusion phase in GCI group (177.47 $\pm 32.4 \%)$ was significantly higher than that in HTEA group $(138.45 \pm 27.2 \%)(P<0.05)$. The hyperperfusion was followed by at least 2 hours of hypoperfusion in GCI group, but not significant in HTEA group. At the most time points of hypoperfusion, the CBF in GCI group was lower than that in sham and HTEA groups $(P<0.05)$.

\section{Mortality and Neurodeficit Scores}

The mortality in the sham group was $0 \%(0 / 18)$, in the GCI group, it was $15.38 \%(4 / 26)$ at $24 \mathrm{~h}$ and $41.67 \%(5 / 12)$ at $72 \mathrm{~h}$. And in the HTEA group, 3 rats were excluded because 1 catheter was not in the epidural space by autopsy and EEG of 2 rats were not isoelectric, the mortality was $12.05 \%(3 / 24)$ and $30.00 \%(3 / 10)$ at $24 \mathrm{~h}$ and $72 \mathrm{~h}$, respectively. There was no significant difference in mortality among the groups (Fig. 2A). Compared with the sham group, the Neurodeficit Scores in both the GCI and the HTEA groups were much lower $(P<0.05)$ (Fig. 2B). The HTEA group demonstrated higher NDS compared with the GCI group both at $24 \mathrm{~h}(67.3 \pm 5.85$ vs. $59.1 \pm 5.53 ; P<0.05)$ and $72 \mathrm{~h}(69.0 \pm 4.20$ vs. $61.9 \pm 5.15 ; P<0.05)$. 
Fig. 1. CBF during Ischemia and Reperfusion. CBF was displayed as percentage of baseline in the four groups $(n=4)$. There were no significant changes of $\mathrm{CBF}$ in sham group and sham-HTEA group animals throughout the experiment. After global cerebral ischemia, HTEA decreased the $\mathrm{CBF}$ at the hyperperfusion stage $(P<0.01)$ and improved the followed hypoperfusion $(P<0.05)$. CBF: cerebral blood flow; HTEA: high thoracic epidural anesthesia; GCI: global cerebral ischemia.* $P<0.05$ vs Sham; \# $P<0.05$ vs GCI group; ** $P<0.01$ vs. Sham group.
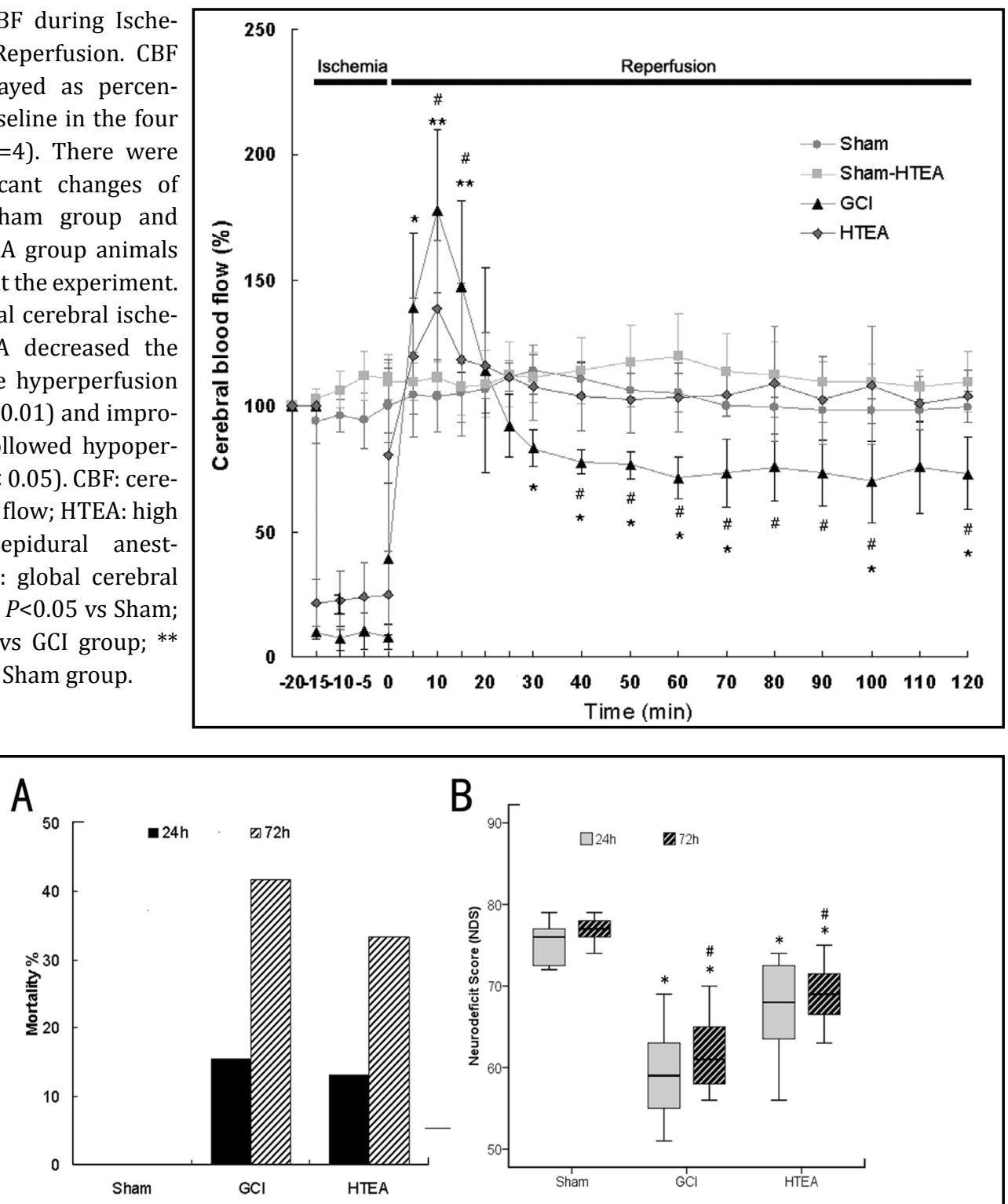

Fig. 2. Mortality and Neurodeficit Scores. (A) The mortality among the sham, GCI and HTEA groups were not statistically significant at $24 \mathrm{~h}$ or $72 \mathrm{~h}$ after ischemia. (B) Neurodeficit Scores (NDS) was presented as median and 25th-75th percentile at $24 \mathrm{~h}$ (sham, $\mathrm{n}=11$; GCI, $\mathrm{n}=13$; HTEA, $\mathrm{n}=11$ ) and $72 \mathrm{~h}$ ( $\mathrm{n}=7$ per group). Significant differences were observed among the three groups at $24 \mathrm{~h}$ and $72 \mathrm{~h}$ after ischemia. HTEA: high thoracic epidural anesthesia; GCI: global cerebral ischemia; NDS: Neurodeficit Scores. ${ }^{*} P<0.05$ vs Sham; \# $P<0.05$ vs GCI group.

\section{Nissl Staining}

Nissl histology revealed delayed cell death characteristics at $72 \mathrm{~h}$ in this study. In the CA1 region of the sham group, the cells had abundant cytoplasm and Nissl bodies, and the cell outlines were clear. In the GCI group, the cells were arranged sparsely, and the cell outlines were fuzzy. The number of cells with eumorphism in the HTEA group was more than those in the GCI group (Fig. 3A). Quantitative cell counts revealed that the number of surviving CA1 neurons at $72 \mathrm{~h}$ (Fig. 3C). The number of surviving neurons in the HTEA group was lower than that in the sham group $(146.8 \pm 14.82$ neurons/mm vs. $169.5 \pm 18.44$ neurons $/ \mathrm{mm}$; $P<0.01$ ), and the number in the HTEA group was increased 3 -fold in comparison to that in the GCI group (146.8 \pm 14.82 neurons $/ \mathrm{mm}$ vs. $48.2 \pm 10.06$ neurons $/ \mathrm{mm} ; P<0.01$ ). 
Fig. 3. Nissl and TUNEL staining of neurons in the hippocampal CA1 region. Nissl staining (A) and the bar graph (C) present the cell counts of surviving neurons at $72 \mathrm{~h}$ after reperfusion. Scale bar represents 30 $\mu \mathrm{m}$. Photomicrographs of TUNEL preparations (B) and the quantitative analysis (D) showed the number of TUNELpositive neurons. Scale bar represents $100 \mu \mathrm{m}$. HTEA markedly decreased the loss of pyramidal cells and the number of apop-
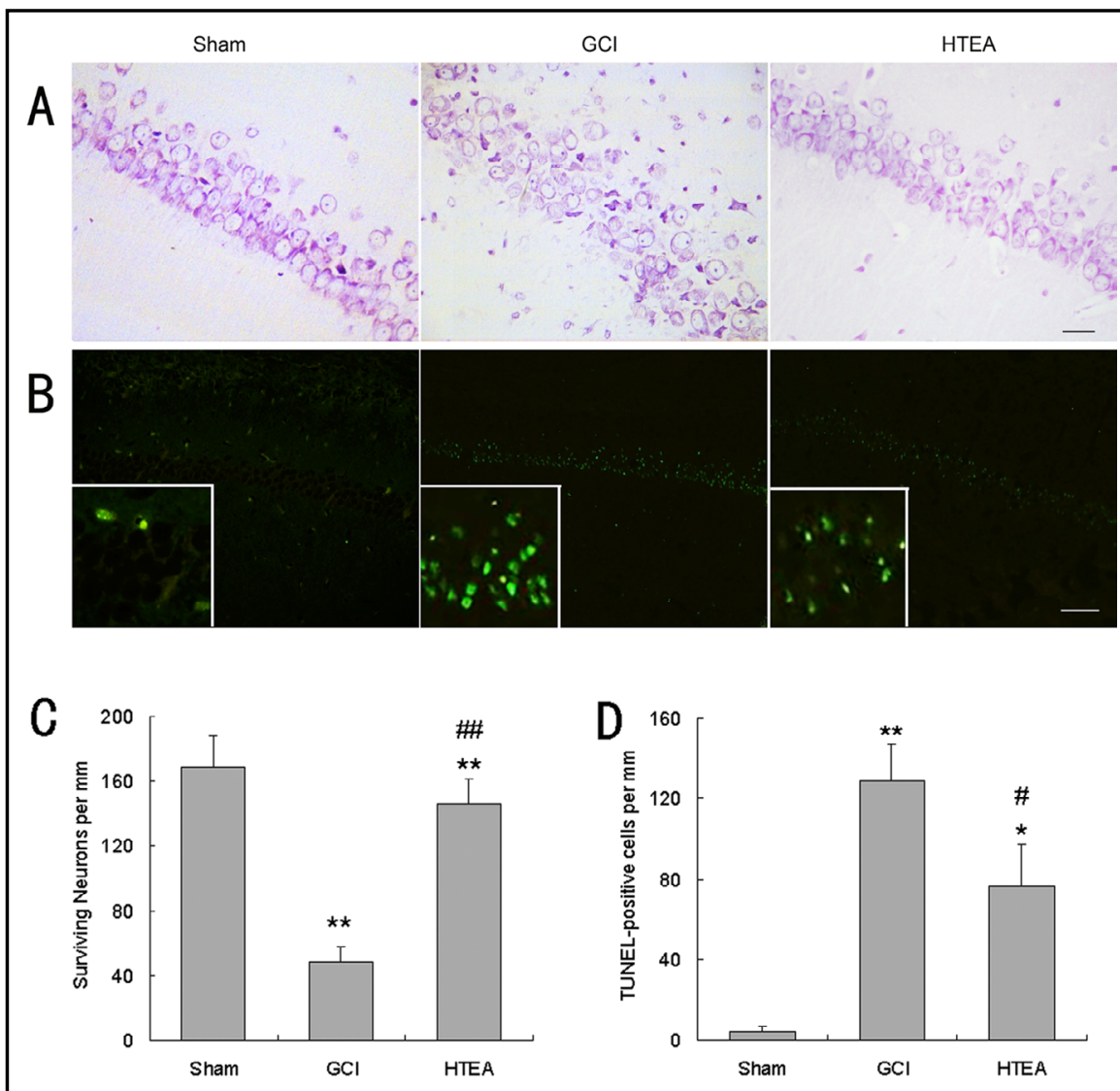

totic neurons in this hippocampal CA1 region ( $\mathrm{n}=4)$. HTEA: high thoracic epidural anesthesia; GCI: global cerebral ischemia. ${ }^{*} P<0.05$ vs. Sham; \# $P<0.05$ vs. GCI group; ${ }^{* *} P<0.01$ vs. Sham group; \#\# $P<0.01$ vs. GCI group.

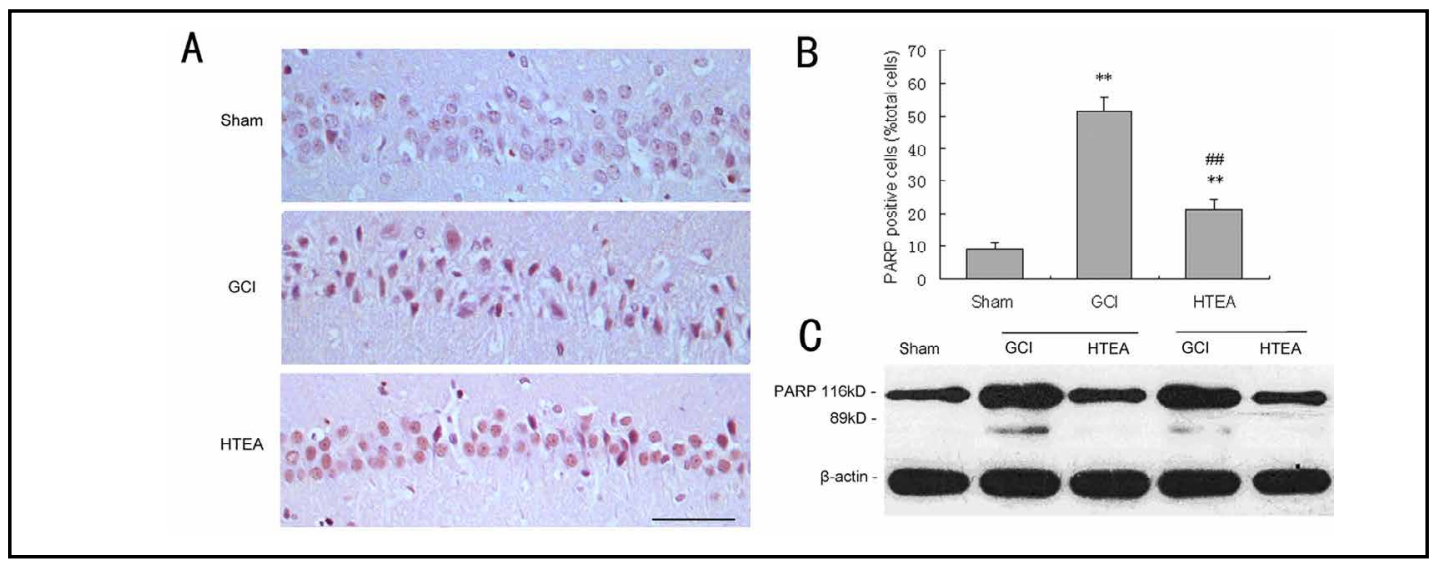

Fig. 4. The activation of PARP expression induced by global cerebral ischemia. (A) Immunohistochemistry staining of PARP p116 are shown at $24 \mathrm{~h}$. Scale bar represents $100 \mu \mathrm{m}$. (B) The percent of PARP positive cells of the immunohistochemistry staining is expressed as mean $\pm S D$ (sham, $n=5$; GCI, $n=7$; HTEA, $n=5$ ). The percent in the HTEA group was significantly lower than that of the GCI group. (C) Western blot analysis of PARP levels in the hippocampus at $24 \mathrm{~h}$ and $72 \mathrm{~h}$ after global cerebral ischemia. Significant decreases were present in PARP p116 in the HTEA group, or no band of PARP p89 was detected in the HTEA group at both time points $(\mathrm{n}=3)$. HTEA: high thoracic epidural anesthesia; GCI: global cerebral ischemia. ${ }^{* *} P<0.01$ vs. Sham group; \#\# $P<0.01$ vs. GCI group. 


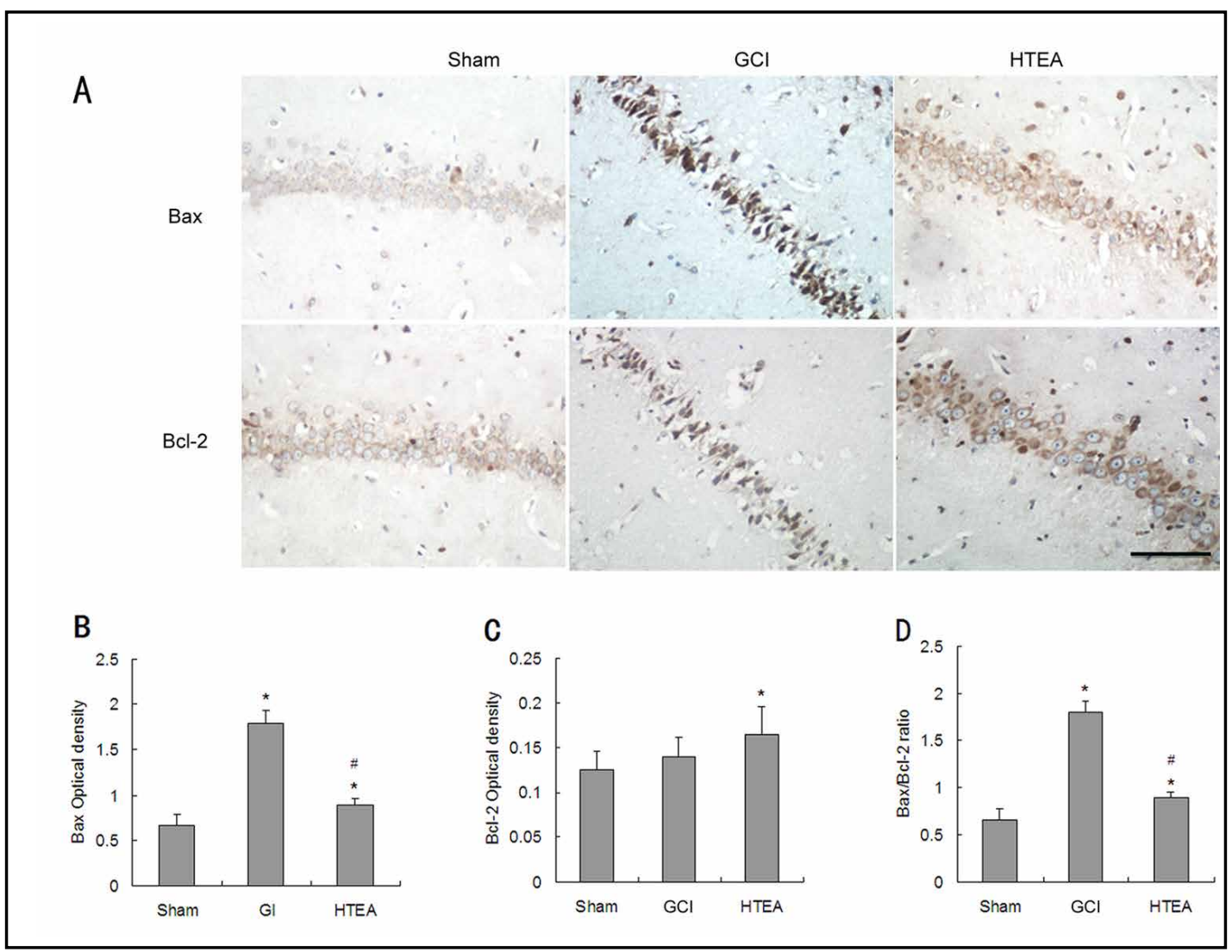

Fig. 5. Immunohistochemistry staining of Bax and Bcl-2. (A) The expression of Bax and Bcl-2 in the hippocampal CA1 region at $24 \mathrm{~h}$ after reperfusion are shown by immunohistochemistry staining. Scale bar represents $100 \mu \mathrm{m}$. (B-D) The OD of Bcl-2, Bax and the Bax/Bcl-2 ratio. HTEA markedly tended to downregulate the Bax expression, and upregulate the Bcl-2 expression (sham, n=5; GCI, n=7; HTEA, n=5). Therefore, the $\mathrm{Bax} / \mathrm{Bcl}-2$ ratio was obviously lower in the HTEA group, compared with GCI group, but it was still higher than that in the sham group. HTEA: high thoracic epidural anesthesia; GCI: global cerebral ischemia. OD: optical density. * $P<0.05$ vs. Sham group; \# $P<0.05$ vs. GCI group.

\section{TUNEL staining}

There were few TUNEL-positive cells in the sham group. A large number of TUNELpositive neurons in the CA1 region were observed in the GCI group rats at $72 \mathrm{~h}$ after global ischemia. In contrast, decreased TUNEL was observed in the HTEA group, compared with the GCI group (Fig. 3B). Cell counting (Fig. 3D) showed that HTEA reduced the number of TUNEL-positive cells by $44.58 \%$ (71.73 \pm 18.05 neurons/mm vs. $129.43 \pm 19.46$ neurons/ $\mathrm{mm} ; P<0.05)$.

\section{PARP Protein Expression}

In immunohistochemical staining, slight expression of PARP was observed in the hippocampal CA1 region in the sham group rats, and strong nuclear immunostaining as well as moderate cytoplasmic staining were increased at $24 \mathrm{~h}$ after ischemia. In contrast, the expression was downregulated in the HTEA group (Fig. 4A). The percent of PARP positive cells in the GCI group was significantly increased compared with that in the sham group ( $51.25 \pm 4.44 \%$ vs. $9.48 \pm 1.70 \%$; $P<0.01)$. Compared with the GCI group, HTEA lead to a significant decrease in the percent $(51.25 \pm 4.44 \%$ vs. $21.12 \pm 3.06 \% ; P<0.01)$ (Fig. 4B). Western blot analysis demonstrated a consistent amount of $\beta$ - actin in all of the groups. However, an increase in PARP p116 (116 kD) and a weak band of PARP p89 (89 kD) were 


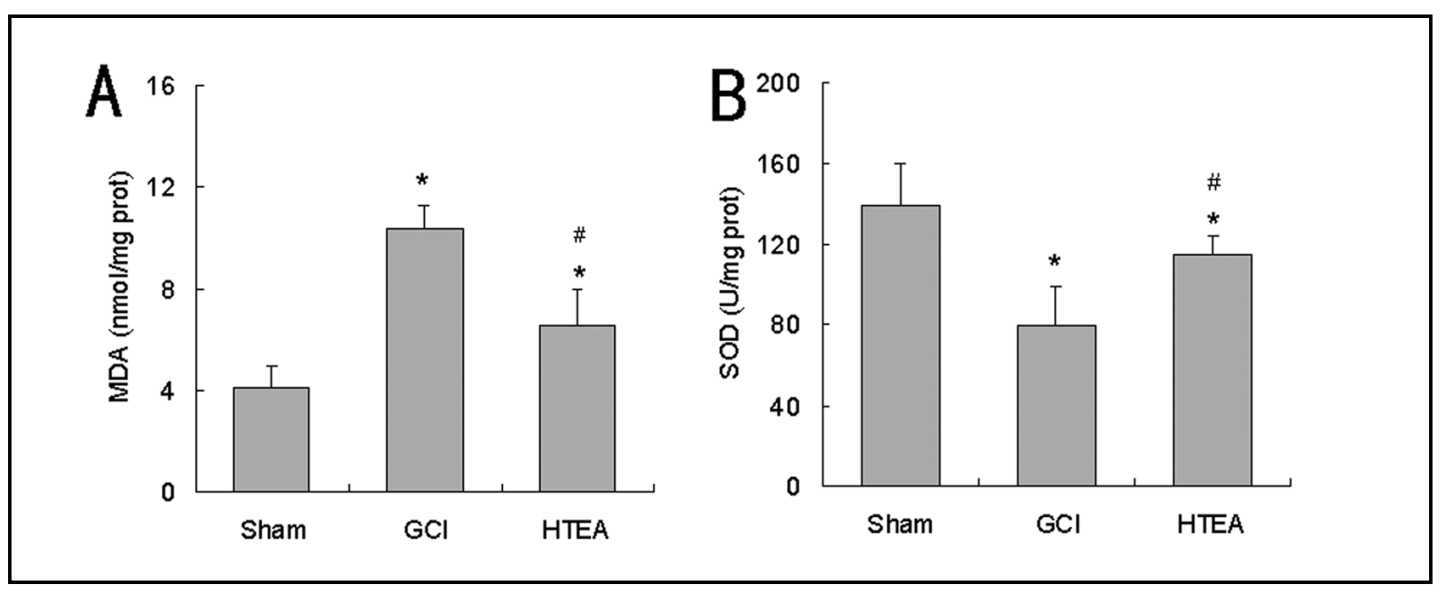

Fig. 6. The hippocampal MDA and SOD levels. HTEA significantly reduced MDA concentrations (A) and increased SOD levels (B) in the hippocampus $24 \mathrm{~h}$ after reperfusion $(\mathrm{n}=6)$. HTEA: high thoracic epidural anesthesia; GCI: global cerebral ischemia; MDA: malondialdehyde; SOD: superoxide dismutase. ${ }^{*} P<0.05$ vs. Sham group; \# $P<0.05$ vs. GCI group.

detected at $24 \mathrm{~h}$ and $72 \mathrm{~h}$ after reperfusion in the GCI groups. There was no PARP p89 expression and a decrease in PARP p116 in the HTEA group, in comparison to that in the GCI group, at both time points (Fig. 4C).

Immunohistochemical Staining of Bcl-2 and Bax

The results of immunohistochemical staining at $24 \mathrm{~h}$ after ischemia are shown in Fig. 3. Bcl-2- and Bax-positive products were brown and granular and were mainly distributed in the cytomembrane and the cytoplasm of pyramidal neurons (Fig. 5A). Bcl-2 and Bax showed a weak positive reaction in the sham group rats. After global ischemia simulation, the expression of Bax increased significantly at $24 \mathrm{~h}$ after ischemia $(P<0.05)$, and this increase was much greater than that in the HTEA group $(P<0.05)$. After HTEA therapy, Bcl-2-positive cells in the cytoplasm of hippocampal CA1 neurons were increased compared with the sham group $(P<0.05)$, and there was no significant difference between the HTEA and GCI groups (Fig. 5B-C). Therefore, a marked enhancement of the Bax/Bcl-2 ratio was observed in the GCI group, compared with the sham group $(P<0.05)$, and then an obvious decline in this ratio occurred after HTEA treatment $(P<0.05)$ (Fig. 5D).

\section{MDA Concentration and SOD Activity}

In the GCI and HTEA groups, MDA concentrations in the hippocampus were significantly higher than those in the sham group $(P<0.05)$, and SOD activities were significantly lower than those in the sham group $(P<0.05)$. Compared with GCI group, HTEA significantly reduced MDA concentrations and increased SOD levels of the hippocampus $(P<0.05)$ (Fig. 6).

\section{Discussion}

In the present study, we first identified that continuous HTEA $0.5 \%$ bupivacaine reduced apoptotic neurons in the CA1 of the hippocampus and the NDS were improved at $72 \mathrm{~h}$ after global cerebral ischemia/reperfusion. We further found that HTEA tended to reduce the activation of PARP and the expression of Bax proteins with a decrease of the Bax/ $\mathrm{Bcl}-2$ ratio at $24 \mathrm{~h}$ after reperfusion. And this neuroprotective effect may be partly due to improved CBF disturbance and reduced oxidative stress. 
Consistent with these reports, severe damage of the CA1 pyramidal neurons was detectable at $72 \mathrm{~h}$ after reperfusion [28, 31]. Consequently, global cerebral ischemia resulted in disorders of motor and sensory function [30]. We found that HTEA reduced GCIinduced apoptosis in the hippocampal CA1 region, and it improved NDS at $24 \mathrm{~h}$ and $72 \mathrm{~h}$ after reperfusion. Although the mortality rate was not reduced, consistent with the previous report [22], we could still observe the beneficial effects of HTEA, which points towards a higher number of surviving CA1 neurons and a better neurologic outcome.

Neurotoxicity and hypotension caused by local anesthetics intrathecal injection are the well-known side effects, which are dependent upon the amount and concentration of local anesthetic agents. As described in previous studies, $0.5 \%$ bupivacaine did not induce apoptosis in rat cortical astrocytes and has no neurotoxic damage [32, 33]. And in the $0.5 \%$ bupivacaine $15-20 \mu \mathrm{l} / \mathrm{h}$ of TEA model, cardiorespiratory depression or extensive motor blockade were not observed in adult rats [7,34].

On the other hand, due to the differential sensitivity of the preganglionic sympathetic fibers compared to the somatic A $\delta$ and C fibers, it is generally assumed that sympathetic B fibers are blocked whenever a sensory block exists. Continuous TEA using $15 \mu \mathrm{L} / \mathrm{h}$ bupivacaine $0.5 \%$ in the thoracic segments indicated the blocked of sympathetic preganglionic neurons in the thoracic spinal cord from Th1 down to segment Th13, and a motor blockade of the front paws occurred during infusion of only $30 \mu \mathrm{L} /$ hour bupivacaine $0.5 \%$ [35]. Although we did not measure thoracic sympathetic activity in this paper, we observed loss of pain sensation in the thoracic area, which implied that the sympathetic nerve fibers supplying the head (T1-4) could be blocked.

Previous reports indicated that during stimulation of superior cervical ganglion plus contralateral nerve section, local cerebral blood flow was reduced on the stimulated side [36]. Sympathetic ganglionic blockade with hexamethonium $[37,38]$ produce sympatholytic effects and decrease plasma catecholamine activity, improve neurologic outcome from cerebral ischemia. In contrast, sympathetic stimulation with physostigmine [39] worsens ischemic outcome by a mechanism that is associated with increases in plasma epinephrine and norepinephrine. In addition, the study of T2 sympathectomy [40], performing measurements 2-4 weeks after intervention, showed increased the blood flow volume, rate and the vascular diameter in the common carotid artery, and the same effects were observed from 5 minutes after performing SGB or HTEA [41]. Accordingly, the neuropreotective effect of HTEA might be associated with the CBF changes after thoracic sympathetic block.

It is observed that global cerebral ischemia is followed first by a short phase of postischemic hyperemia that emerges into a secondary prolonged phase of delayed hypoperfusion [8, 9]. Both hyperperfusion and hypoperfusion do harm to the recovery of the ischemic brain. A high CBF in the late hyperperfusion phase correlated with a low number of surviving cortical neurons 7 days after global cerebral ischemia, which is presumably accompanied by an enhanced generation of oxygen-derived free radicals and therefore increased neuronal death [42]. Delayed hypoperfusion after global cerebral ischemia is a pathophysiological process with progressively increasing precapillary arteriolar tone and consequent breakdown of the capillary microcirculation, and reversal of delayed hypoperfusion should be part of a therapeutic strategy to alleviate secondary brain injury after stroke [9]. In this study, we observed that HTEA decrease hyperperfusion and improve at least 2 hours of hypoperfusion after reperfusion, perhaps helping to decrease the overproduction of free radicals and attenuate the cerebral microvascular resistance. However, under resting conditions in health, there was little tonic sympathetic activity, and cerebral sympathetic denervation did not change CBF in healthy rats, which is consistent with previous studies [19].

In 4-vessel occluded rats, there were still collateral vessels probably originate from cervical branches of the subclavian arteries to the brain, and blood flow from the anterior spinal artery to the brainstem and cerebellum [24]. In newborn piglets, after superior cervical sympathetic ganglionectomy, denervated CBF during asphyxia was higher compared with innervated CBF [43]. However, in stroke-prone spontaneously hypertensive rats, there was 
no significant difference in CBF between the innervated and denervated animals (bilateral superior cervical ganglionectomy) during ischemia [44]. In our study, the CBF of HTEA group rats had a higher trend compared with that of GCI group rats during ischemia period, but without statistical significance. Not significant may be due to the small sample size, a doubled CBF sample size might be very well relevant. If any, it is presumed that the increased blood flow under HTEA occurred not only at the hypoperfusion stage after reperfusion, but also in the ischemic period.

Interestingly, ROS production is highly relevant to the vascular response to ischemia. The superoxide radicals in hippocampal CA1 neurons returned to the basal level after 3 days in the global cerebral ischemia model, but in the SOD1 transgenic rats, the complete return of the superoxide radical level to the basal level within 1 day correlates well with the rapid normalization of CBF within 1 day [45]. SOD 1 overexpression confers protection by blocking the caspase activation in the mitochondrial signaling pathway of apoptotic cell death in hippocampal CA1 neurons after global ischemia [2]. In addition, lipid peroxidation due to ischemia reperfusion injury is among the primary causes of tissue injury. MDA is an intermediate product of lipid peroxidation and is used for the assessment of tissue injury related to lipid peroxidation [7, 31]. In the present study, HTEA was demonstrated to inhibit MDA production and increase the antioxidant enzyme of SOD for scavenging oxygen-free radicals, suggesting that the inhibition of oxidative stress may be partly due to the blockade of the sympathetic nerve distributed to the brain.

The mitochondrial-mediated pathway of apoptosis is regulated by the Bcl-2 family of antiapoptotic and proapoptotic proteins, which play a crucial role in intracellular apoptotic signal transduction by regulating permeability of the mitochondrial membrane [2]. The Bax/ Bcl-2 ratio is a measure of a cell's vulnerability to apoptosis, and the ratio may be more important than either promoter alone in determining apoptosis [46]. In the downstream mitochondrial pathway of apoptosis, activated caspase-3, recognizing the structure of PARP, generates two proteolytic products: a $29 \mathrm{kD}$ and a $89 \mathrm{kD}$ fragments [47], which is considered a hallmark of apoptosis [5]. During global cerebral ischemia, extensive activation of PARP can rapidly result in cell death through depletion of energy stores because four molecules of adenosine triphosphate (ATP) are consumed for NAD regeneration [4]. Inhibiting PARP attenuates cerebral vasospasm after subarachnoid hemorrhage in rabbits [48], and the PARP inhibitor treatment decreases the number of apoptotic neurons and improves the neuronal survival and memory during GCI [49]. Our previous study had reported that the expression of PARP p89 after GCI was attenuated under HTEA injected by $0.125 \%$ bupivacaine [50]. In the present study, although the expression of Bcl-2 protein had no significant increase under HTEA, the decreased expression of Bax protein and the decreased Bax/Bcl-2 ratio were observed. And we also observed low expression of uncleaved PARP p116 and little PARP p89 cleavage fragment. Thus, the less activation of the mitochondrial signaling pathway of apoptosis may be involved in HTEA-induced neuroprotective effect in cerebra ischemia injury.

It should be noted that the present study might have limitations. Firstly, an additional group, receiving systemic bupivacaine would have to be included, to exclude the possible systemic effects of bupivacaine. It has been reported that bupivacaine decreased local cerebral glucose utilization [51] and block voltage-dependent sodium channels, and consequently have a neuroprotective action against ischemic insult in the central nervous system[52, 53], which is most likely due to the drug's systemic effect rather than deafferentation. Secondly, further studies are needed to compare the beneficial effects of bupivacaine and other local anesthetics injected into the high thoracic epidural space. Thirdly, whether the neuroprotective effect can be transferred to other animal models or even humans needs further explored. The mechanism of the functional connection between the thoracic cord and the hippocampus is not fully understood, although the reduced number of apoptotic neurons with HTEA after global cerebral ischemia is observed.

In conclusion, continuous HTEA plays roles in attenuating hippocampal apoptosis after global cerebral ischemia. These protective effects may be partly due to the improved 
microcirculation, the reduced oxidative stress, the decreased Bax/Bcl-2 ratio and the less activation of PARP.

\section{Abbreviations}

HTEA (high thoracic epidural anesthesia); GCI (global cerebral ischemia); NDS (neurodeficit scores); PARP (poly (ADP-ribose) polymerase); SOD (superoxide dismutase); MDA (malondialdehyde); CA1 (cornu ammonis area 1); ROS (reactive oxygen species); SGB (stellate ganglion block); OD (optical density).

\section{Acknowledgments}

The authors express our sincere thanks to Hongbo Jin, Jing Ma, Ruibo Zhao and Xiaomei Li for their technical assistance. This study was supported by Wu Jieping special fund no. 320.6250.12262, Heilongjiang Provincial Natural Science Foundation D201071, and the Hospital Fund, no. JJZ2011-09.

\section{References}

1 Liang HW, Qiu SF, Shen J, Sun LN, Wang JY, Bruce IC, Xia Q: Genistein attenuates oxidative stress and neuronal damage following transient global cerebral ischemia in rat hippocampus. Neurosci Lett 2008;438:116-120.

- Sugawara T, Fujimura M, Noshita N, Kim GW, Saito A, Hayashi T, Narasimhan P, Maier CM, Chan PH: Neuronal death/survival signaling pathways in cerebral ischemia. NeuroRX 2004;1:17-25.

-3 Nitatori T, Sato N, Waguri S, Karasawa Y, Araki H, Shibanai K, Kominami E, Uchiyama Y: Delayed neuronal death in the CA1 pyramidal cell layer of the gerbil hippocampus following transient ischemia is apoptosis. J Neurosci 1995;15:1001-1011.

4 Takahashi K, Pieper aa, Croul SE, Zhang J, Snyder SH, Greenberg JH: Post-treatment with an inhibitor of poly(ADP-ribose) polymerase attenuates cerebral damage in focal ischemia. Brain Res 1999;829:46-54.

5 Lindahl T, Satoh MS, Poirier GG, Klungland A: Post-translational modification of poly(ADP-ribose) polymerase induced by DNA strand breaks. Trends Biochem Sci 1995;20:405-411.

-6 Saito A, Maier CM, Narasimhan P, Nishi T, Song YS, Yu F, Liu J, Lee YS, Nito C, Kamada H, Dodd RL, Hsieh LB, Hassid B, Kim EE, Gonzalez M, Chan PH: Oxidative stress and neuronal death/survival signaling in cerebral ischemia. Mol Neurobiol 2005;31:105-116.

7 Bedirli N, Akyurek N, Kurtipek O, Kavutcu M, Kartal S, Bayraktar AC: Thoracic epidural bupivacaine attenuates inflammatory response, intestinal lipid peroxidation, oxidative injury, and mucosal apoptosis induced by mesenteric ischemia/reperfusion. Anesth Analg 2011;113:1226-1232.

-8 Miller CL, Lampard DG, Alexander K, Brown WA: Local cerebral blood flow following transient cerebral ischemia. I. Onset of impaired reperfusion within the first hour following global ischemia. Stroke 1980;11:534-541.

-9 Hauck EF, Apostel S, Hoffmann JF, Heimann A, Kempski O: Capillary flow and diameter changes during reperfusion after global cerebral ischemia studied by intravital video microscopy. J Cereb Blood Flow Metab 2004;24:383-391.

10 Gupta MM, Bithal PK, Dash HH, Chaturvedi A, Mahajan RP: Effects of stellate ganglion block on cerebral haemodynamics as assessed by transcranial Doppler ultrasonography. Br J Anaesth 2005;95:669-673.

11 Westerhaus MJ, Loewy AD: Central representation of the sympathetic nervous system in the cerebral cortex. Brain Res 2001;903:117-127.

12 Murata Y, Shibata H, Chiba T: A correlative quantitative study comparing the nerve fibers in the cervical sympathetic trunk and the locus of the somata from which they originate in the rat. J Auton Nerv Syst 1982;6:323-333. 


\section{Cellular Physiology $\quad$ Cell Physiol Biochem 2014;34:1227-1240 and Biochemistry

13 Higa K, Hori K, Harasawa I, Hirata K, Dan K: High thoracic epidural block relieves acute herpetic pain involving the trigeminal and cervical regions: comparison with effects of stellate ganglion block. Reg Anesth Pain Med 1998;23:25-29.

14 Kim EM, Yoon KB, Lee JH, Yoon DM, Kim do H: The effect of oxygen administration on regional cerebral oxygen saturation after stellate ganglion block on the non-blocked side. Pain Physician 2013;16:117-124.

15 Lang 0, Svoboda L, Balon HR: Effect of sympathetic blockade on cerebral perfusion demonstrated on Tc99m HMPAO SPECT. J Neurol 2002;249:108-109.

-16 Chun-jing H, Shan 0, Guo-dong L, Hao-xiong N, Yi-ran L, Ya-ping F: Effect of cervical sympathetic block on cerebral vasospasm after subarachnoid hemorrhage in rabbits. Acta Cir Bras 2013;28:89-93.

-17 Treggiari MM, Romand JA, Martin JB, Reverdin A, Rufenacht DA, de Tribolet N: Cervical sympathetic block to reverse delayed ischemic neurological deficits after aneurysmal subarachnoid hemorrhage. Stroke 2003;34:961-967.

-18 Kang CK, Oh ST, Chung RK, Lee H, Park CA, Kim YB, Yoo JH, Kim DY, Cho ZH: Effect of stellate ganglion block on the cerebrovascular system: magnetic resonance angiography study. Anesthesiology 2010;113:936-944.

19 ter Laan M, van Dijk JM, Elting JW, Staal MJ, Absalom AR: Sympathetic regulation of cerebral blood flow in humans: a review. Br J Anaesth 2013;111:361-367.

20 Sandor P: Nervous control of the cerebrovascular system: doubts and facts. Neurochem Int 1999;35:237259.

21 Xiong L, Wang Y, Wang Q Zhou Q: Cervical sympathetic trunk transection affects inducible nitric oxide synthase expression in rat hippocampus following focal cerebral ischemia/reperfusion injury. Neural Regen Res 2008;3:1084-1087.

-22 Kozian A, Schilling T, Hachenberg T: Non-analgetic effects of thoracic epidural anaesthesia. Curr Opin Anesthesiol 2005;18:29-34.

-23 Guo Z, Wang JP: Blockade of spinal nerves attenuates myocardial apoptosis in acute myocardial ischaemia/ infarction in rats. Eur J Anaesthesiol 2010;27:146-152.

-24 Pulsinelli WA, Brierley JB: A new model of bilateral hemispheric ischemia in the unanesthetized rat. Stroke 1979;10:267-272.

25 Yoshizumi H, Fujibayashi Y, Kikuchi H: A new approach to the integrity of dual blood-brain barrier functions of global ischemic rats. Barrier and carrier functions. Stroke 1993;24:279-284.

26 Ostrowski RP, Colohan AR, Zhang JH: Mechanisms of hyperbaric oxygen-induced neuroprotection in a rat model of subarachnoid hemorrhage. J Cereb Blood Flow Metab 2005;25:554-571.

27 Martinez G, Musumeci G, Loreto C, Carnazza ML: Immunohistochemical changes in vulnerable rat brain regions after reversible global brain ischaemia. J Mol Histol 2007;38:295-302.

28 Cheng O, Ostrowski RP, Wu B, Liu W, Chen C, Zhang JH: Cyclooxygenase-2 mediates hyperbaric oxygen preconditioning in the rat model of transient global cerebral ischemia. Stroke 2011;42:484-490.

29 Duan YL, Wang SY, Zeng QW, Su DS, Li W, Wang XR, Zhao Z: Astroglial reaction to delta opioid peptide [D-Ala2, D-Leu5] enkephalin confers neuroprotection against global ischemia in the adult rat hippocampus. Neuroscience 2011;192:81-90.

-30 Geocadin R, Ghodadra R, Kimura T, Lei H, Sherman D, Hanley D, Thakor N: A novel quantitative EEG injury measure of global cerebral ischemia. Clin Neurophysiol 2000;111:1779-1787.

-31 Ji Q, Hui K, Zhang L, Sun X, Li W, Duan M: The effect of hydrogen-rich saline on the brain of rats with transient ischemia. J Surg Res 2011;168:e95-101.

-32 Lee WY, Park CJ, Shin TJ, Yum KW, Yoon TG, Seo KS, Kim HJ: Only tetracaine and not other local anaesthetics induce apoptosis in rat cortical astrocytes. Br J Anaesth 2009;103:719-725.

33 Takenami T, Yagishita S, Nara Y, Tsai YH, Hiruma H, Kawakami T, Hoka S: Spinal procaine is less neurotoxic than mepivacaine, prilocaine and bupivacaine in rats. Reg Anesth Pain Med 2009;34:189-195.

-34 Freise H, Lauer S, Konietzny E, Hinkelmann J, Minin E, Van Aken HK, Lerch MM, Sielenkaemper AW, Fischer LG: Hepatic effects of thoracic epidural analgesia in experimental severe acute pancreatitis. Anesthesiology 2009;111:1249-1256.

-35 Freise H, Anthonsen S, Fischer LG, Van Aken HK, Sielenkamper AW: Continuous thoracic epidural anesthesia induces segmental sympathetic block in the awake rat. Anesth Analg 2005;100:255-262.

-36 Tuor UI: Local distribution of the effects of sympathetic stimulation on cerebral blood flow in the rat. Brain Res 1990;529:224-231. 


\section{Cellular Physiology $\quad$ Cell Physiol Biochem 2014;34:1227-1240 and Biochemistry \\ Li et al.: HTEA in Hippocampal Apoptosis after GCI in Rats}

-37 Shu CC, Hoffman WE, Thomas C, Albrecht RF: Sympathetic activity enhances glucose-related ischemic injury in the rat. Anesthesiology 1993;78:1120-1125.

-38 Werner C, Hoffman WE, Thomas C, Miletich DJ, Albrecht RF: Ganglionic blockade improves neurologic outcome from incomplete ischemia in rats: partial reversal by exogenous catecholamines. Anesthesiology 1990;73:923-929.

39 Schultz JA, Hoffman WE, Albrecht RF: Sympathetic stimulation with physostigmine worsens outcome from incomplete brain ischemia in rats. Anesthesiology 1993;79:114-121.

$>40$ Jeng JS, Yip PK, Huang SJ, Kao MC: Changes in hemodynamics of the carotid and middle cerebral arteries before and after endoscopic sympathectomy in patients with palmar hyperhidrosis: preliminary results. J Neurosurg 1999;90:463-467.

41 Murakawa K, Noma K, Ishida K, Matsuda M, Terashita K, Maeda S, Izumi R: Circulatory effects of stellate ganglion block and high thoracic epidural block. Masui 1994;43:998-1003. (in Japanese)

-42 Soehle M, Heimann A, Kempski O: Postischemic application of lipid peroxidation inhibitor U-101033E reduces neuronal damage after global cerebral ischemia in rats. Stroke 1998;29:1240-1246; discussion 1246-1247.

43 Goplerud JM, Wagerle LC, Delivoria-Papadopoulos M: Sympathetic nerve modulation of regional cerebral blood flow during asphyxia in newborn piglets. Am J Physiol 1991;260:H1575-1580.

-44 Nagao T, Sadoshima S, Ishitsuka T, Kusuda K, Shiokawa O, Ibayashi S, Fujishima M: Effects of acute superior cervical ganglionectomy on cerebral blood flow and metabolism in stroke-prone spontaneously hypertensive rats subjected to cerebral ischaemia. Clin Exp Pharmacol Physiol 1992;19:489-493.

-45 Xu Y, Liachenko SM, Tang P, Chan PH: Faster recovery of cerebral perfusion in SOD1-overexpressed rats after cardiac arrest and resuscitation. Stroke 2009;40:2512-2518.

-46 Wang Y, Liu X, Zhang D, Chen J, Liu S, Berk M: The effects of apoptosis vulnerability markers on the myocardium in depression after myocardial infarction. BMC Med 2013;11:32-32.

-47 Smrcka M, Horky M, Otevrel F, Kuchtickova S, Kotala V, Muzik J: The onset of apoptosis of neurons induced by ischemia-reperfusion injury is delayed by transient period of hypertension in rats. Physiol Res 2003;52:117-122.

48 Satoh M, Date I, Nakajima M, Takahashi K, Iseda K, Tamiya T, Ohmoto T, Ninomiya Y, Asari S: Inhibition of poly(ADP-ribose) polymerase attenuates cerebral vasospasm after subarachnoid hemorrhage in rabbits. Stroke 2001;32:225-231.

49 Plaschke K, Kopitz J, Weigand Ma, Martin E, Bardenheuer HJ: The neuroprotective effect of cerebral poly(ADP-ribose)polymerase inhibition in a rat model of global ischemia. Neurosci Lett 2000;284:109-112.

50 Huo X, Li ZX, Jiang LH, Wang HM, Qu RH, Wang GN: Effects of high thoracic epidural anesthesia on cerebral ischemia-reperfusion injury in rats. Chin J Anesthesiol 2006;26:710-713. (in Chinese)

51 Kuroda Y, Sakabe T, Nakakimura K, Oshita S, Maekawa T, Ishikawa T, Takeshita H: Epidural bupivacaine suppresses local glucose utilization in the spinal cord and brain of rats. Anesthesiology 1990;73:944-950.

52 Marganella C, Bruno V, Matrisciano F, Reale C, Nicoletti F, Melchiorri D: Comparative effects of levobupivacaine and racemic bupivacaine on excitotoxic neuronal death in culture and N-methyl-Daspartate-induced seizures in mice. Eur J Pharmacol 2005;518:111-115.

53 Lee JR, Han SM, Leem JG, Hwang SJ: Effects of intrathecal bupivacaine in conjunction with hypothermia on neuronal protection against transient spinal cord ischemia in rats. Acta Anaesthesiol Scand 2007;51:60-67. 